\title{
Signal settings design problem with an analytical approach: application in an urban area
}

\author{
A. Polimeni ${ }^{1} \&$ A. Vitetta ${ }^{2}$ \\ ${ }^{1}$ DICIEAMA - Dipartimento di Ingegneria Civile, \\ Informatica, Edile, Ambientale e Matematica Applicata, \\ Università degli Studi di Messina, Italy \\ ${ }^{2}$ DIIES - Dipartimento di Ingegneria dell'informazione, \\ delle Infrastrutture e dell'Energia Sostenibile, \\ Università degli Studi Mediterranea di Reggio Calabria, Italy
}

\begin{abstract}
We report an application for designing signals at junctions in an urban area. The problem formulation connects the gradient evaluation with the path choice model, allowing a robust method to evaluate analytically the derivatives in the gradient projection method based on C-Logit model. The application is developed in the central area of Reggio Calabria, designing the signals at some junctions in a system optimum approach. The objective of the procedure is to minimize the energy spend by a vehicle in an urban area with the optimization of the signals at the junction. Keywords: C-Logit assignment, gradient projection algorithm, network design problem, energy consumption.
\end{abstract}

\section{Introduction}

The design problem applied in this paper relates to the optimal signal design at junctions for minimizing the time spend by the road users and the consumption of the energy. The solution method considers the gradient projection algorithm. In recent years, in literature, for the solution of this problem (also called signal setting design problem) many procedures have been proposed [1] 
The first papers $[2,3]$ consider the path choice as rigid (the demand is inelastic at the level of path choice) and each junction as independent from the others (junctions not coordinated). Advancements in this topic are the assumption of an elastic path choice $[4,5]$ or the coordination among the junctions $[6,7]$. There are other works considering jointly the elastic path choice and the junctions synchronization [8-12].

The signal settings problem can be extended to simulate and/or design a transport network in special situations, i.e. when something happen and it is mandatory to evacuate an urban centre [13-15].

The consumption of the energy in road transport refers to the consumption of fuel by cars and cam be also evaluate with a model [1].

The problem formulation provided in this paper considers elastic path choice and synchronization of signals at junctions. The design is performed with a gradient projection algorithm, based on projecting the search direction into the feasibility set of the solutions. An equilibrium assignment, with implicit path choice, is considered in order to evaluate the network performances. The path choice probability is evaluated whit a C-Logit model [17] that allows evaluating it in a closed form. The evaluation in closed form of the path probability is fundamental to computing analytically the derivative in the gradient algorithm.

In the algorithm, the analytical approach in the gradient evaluation allows a reduction in the computation time respect to a numerical approach.

The advancement reported in this paper is relative to the application in a real dimension network for travel time and energy reduction. Real world application of this method could be made to manage a transport network in real time control and in planning process [18].

The paper is structured as follow. In Section 2 the problem formulation is shortly recalled; in Section 3 an application on a real system is reported. Finally, in Section 4 the conclusions are reported.

\section{Problem formulation}

Figure 1 shows the logical flow of proposed procedure. Starting from an initial network configuration (an initial value of the signals at junctions) and considering fixed the users demand at the levels of departure time, distribution and modal split, an equilibrium assignment procedure allows obtaining some performances of the network (link costs and flows, objective function value). Knowing the network performances, the gradient projection algorithm is applied for generating the new network configuration in term of signals in the junctions. This type of algorithm is choice due the constrained nature of the problem. A test on the value of the objective function indicate if the procedure can be stopped or if start another iteration. 


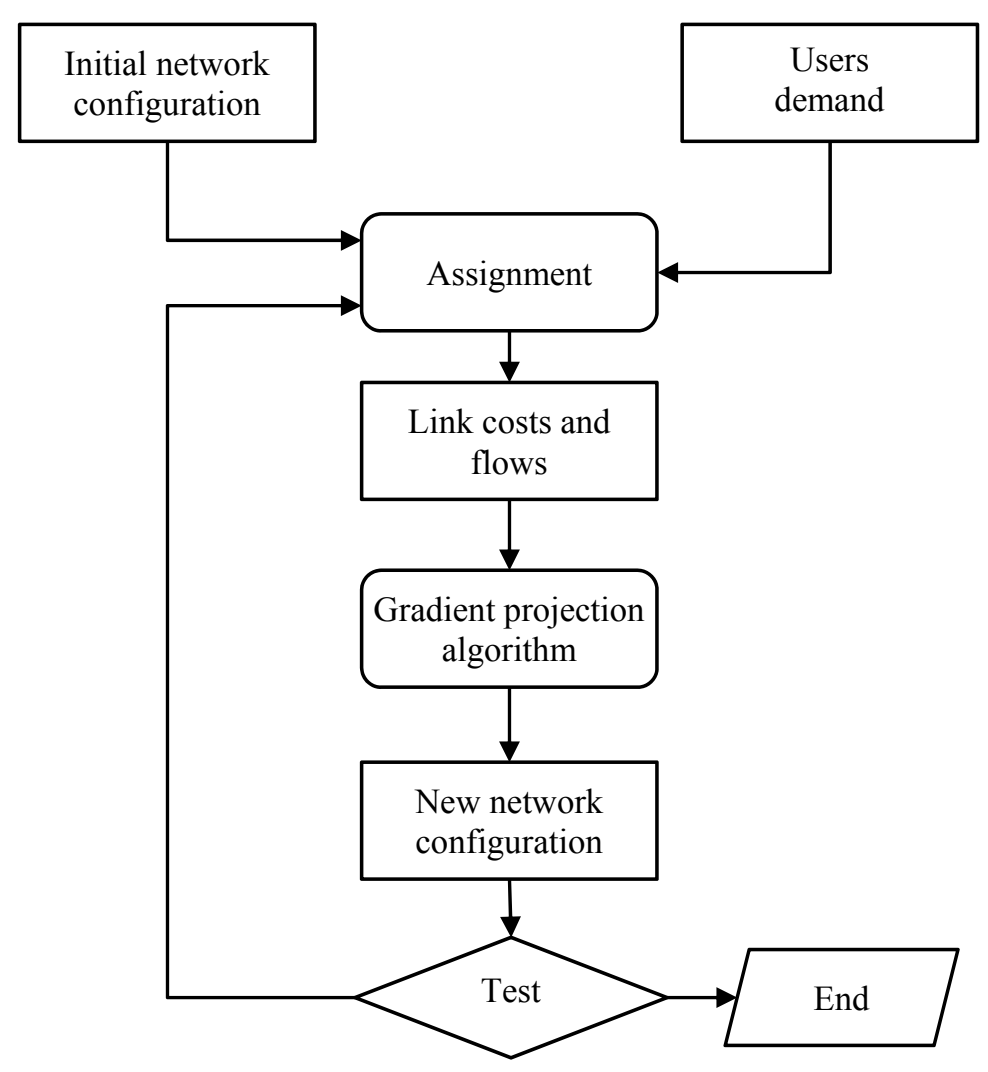

Figure 1: Flowchart of the proposed procedure.

\subsection{Model}

Let $G=(N, L)$ a graph, $N$ is the node set and $L$ is the link set. Each link is relative to one access of the junction. For each link $l$ in $L$ is possible to define the flow $f_{l}$, the link green percentage respect to the cycle length $y_{l}$ and the cost $c_{l}$, defined as a function of the flow and green value:

$$
c_{l}=\gamma_{l}\left(f_{l}, y_{l}\right) \quad \forall l \in L,
$$

where $\gamma_{l}(\cdot)$ is the cost function for $l$, including the running and waiting time at junction. To know more about (1), see [19].

In literature, various are the models proposed to evaluate the cost (i.e. BPR for the running time and Webster function for the waiting time).

The variables above introduced can be inserted into vectors: $\boldsymbol{f}, \boldsymbol{c}, \boldsymbol{\gamma}$ and $\boldsymbol{y}$.

In the following, we suppose that $\boldsymbol{f} \in S_{f,} \boldsymbol{y} \in S_{y}$, where $S_{f}$ is the feasibility set of link flows (the link flows must be not negative and consistent with the path flows); $S_{y}$ is the feasibility set of design variables (the design variables must be not 
negative and less than 1 and consistent with the junction plan).

Considering a path $k$ in the origin-destination pair $o d$, the path probability with the C-Logit model specification is:

$$
q_{k \mid \rho d}(c)=\text { Error! }
$$

with

- $g_{k}$, path cost, obtained as: $g_{k}=g_{k}(\boldsymbol{c})=\Sigma_{l \in k} c_{l}+C F_{k}$;

- $C F_{k}$, Communality Factor (as in [17]), to take into account the overlap among different paths on the same origin destination pair;

- $I_{o d}$, set of all perceived paths between origin destination pair $o d$.

Considering the loading function vector $\varphi$ (with entry $\varphi_{l}$ ), and the Eq. (1) the flow vector $\boldsymbol{f}$ (with entry $f_{l}$ ) is:

$$
f=\varphi(\gamma(f, y))
$$

with

- $\varphi_{l}$, loading function for the link $l$;

- $f_{l}$, link flow, obtained as: $f_{l}=\sum_{o d} d_{o d} \cdot \Sigma_{k \in \text { Iod }} \delta_{l k} \cdot q_{k \mid o d}(\mathbf{c})=\varphi(\mathbf{c})$;

- $d_{o d}$, travel demand between an od pair;

- $\delta_{l k}$, binary variable with value 1 if $l \in k$.

For the signal setting design, it is assumed that the objective is the minimization of the total time $\boldsymbol{z}$ for the users in the system:

$$
\boldsymbol{z}(\boldsymbol{f}, \boldsymbol{y})=(\gamma(\boldsymbol{f}, \boldsymbol{y}))^{T} \cdot \boldsymbol{\varphi}(\gamma(\boldsymbol{f}, \boldsymbol{y}))
$$

Note that in urban area the energy consumption increases with the increase of the total travel time on the network. The optimization of the network configuration in term of capacity in the junctions, for travel time minimization, gives two results:

- the reduction of the congestion (with fixed travel demand, the reduction of the travel time is equivalent to the maximization of the user speed);

- the reduction of the consumption of the energy by the vehicles considering the low speed in congested conditions.

The problem solution is a vector $y$ that minimize the Eq. (4). More details about the model formulation is reported in [20].

\subsection{Solution procedure}

The solution procedure applied to solve problem (4) is the gradient projection algorithm. It is an iterative procedure, based on projecting the search direction into the feasibility set of the solutions. Defined the search direction, two alternatives are available to evaluate the derivative: numerical or analytic approach. Respect to the analytic approach, the numerical approach requires, to guarantee an acceptable approximation of the numerical derivatives, a very small convergence error in assignment. Moreover, the analytical approach requires one assignment procedure for iteration, whereas numerical approach two for iteration. To know more about, see [20]. 
The value of the design vector $\boldsymbol{y}^{i t}$ in an iteration it is equal to the sum of its value in previous iteration $\left(y^{i t-I}\right)$ and an increment. The increment is obtained as the product between the gradient of the objective function (evaluated in the point $\left(f_{\mathrm{SUE}}{ }^{i t}\right.$ (the equilibrium flow), $\boldsymbol{y}^{i t-1}$ ) and the parameter $\mu_{i t}$ (constrained in the range $\left.\left[\mu_{\min }, \mu_{\max }\right]\right)$. The parameter $\mu_{i t}$ is obtained as the optimum of a monodimensional problem as reported in [20].

In this paper, the evaluation of the derivative in the gradient is performed with an analytical approach, possible because the path choice is made with a C-Logit model. Depending on the relationship between the variables $\boldsymbol{y}, \boldsymbol{f}$ and $\boldsymbol{c}$ the solution algorithm can be local convergent (if moving direction is exactly the descent direction) or heuristic (if the descent direction is approximated).

\section{Application}

In this section, an application on a real dimension network is reported. The study area is the town centre of Reggio Calabria (Italy), described in section 3.1.

\subsection{Study area}

Reggio Calabria is a city in the south Italy with a population (in the town centre) of about 180,000 inhabitants. The city is characterized by a longitudinal development, between the coast and the hills and it is the result of reconstruction after the 1908 earthquake. Most of the transport network is formed by one way roads that intersect perpendicularly. In this application, the road network (in Figure 2 a portion of the graph) is represented with 384 links and 188 nodes.

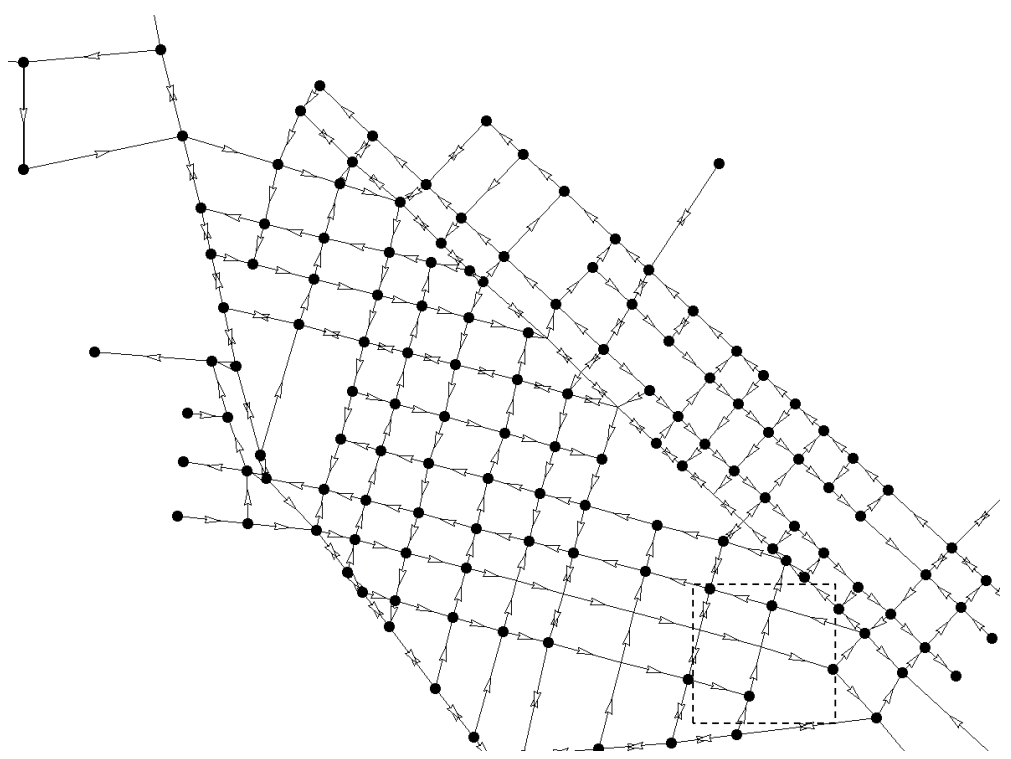

Figure 2: A portion of the Reggio Calabria graph. 


\subsection{Junctions design}

In the network, nine junctions are identified to design the signals (Figure 3a); for each of it the design variable $y_{l}$ must be optimized. In the network other junctions are regulated by traffic light but they are assumed with fixed values. The assumption is that at each junction two phases are designed, in each phase only compatible manoeuvres are permitted. This approach can easily extended to design more than two phases.

Note that (Figure $3 b$ ), considering a link $l$, for each designed variable $y_{l}$ there is a complementary variable $\left(1-y_{l}\right)$ related to the incompatible link. In this way, a subset of link $A$ can be extract from the set $L(A \subseteq L)$.

In Table 1 the optimal value of $\boldsymbol{y}$ are reported, assuming a cycle length equal to 60 seconds and an initial value for design variable equal to 0.5 (including the lost time).

The objective function (4) decrease during the procedure from $6845(\mathrm{~s} \cdot \mathrm{veich} / \mathrm{s})$ to $6161(\mathrm{~s} \cdot$ veich/s). The reduction in the total travel time is about $10 \%$. The result is relevant considering that only about the $5 \%$ of the junction are optimized. The reduction in term of energy consumption (with fixed demand and type of vehicles is about $3 \%$.

The proposed analytical method can be compared with a numerical approach, where the derivatives of function $z()$ are evaluated with the finite element method.

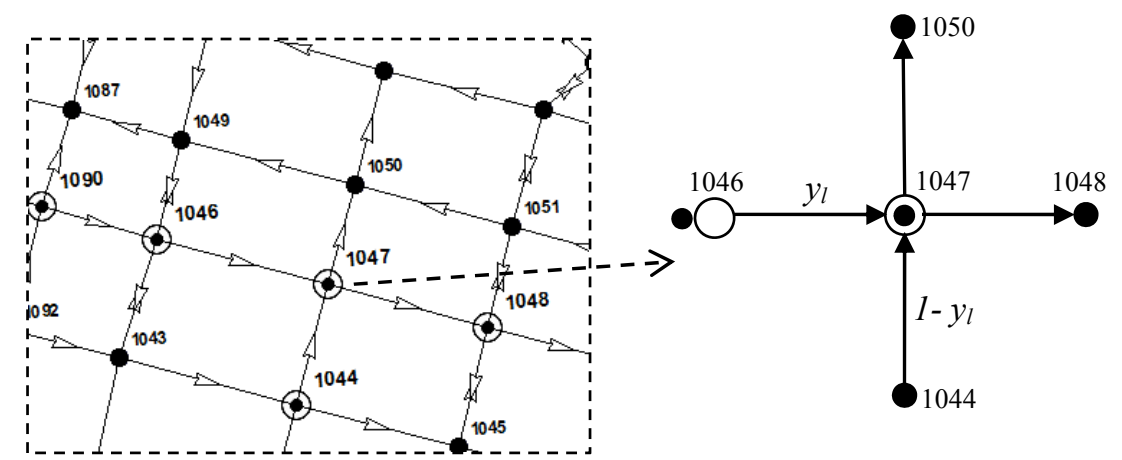

(a)

(b)

Figure 3: (a) Designed junctions and (b) design variables.

To evaluate the derivative of function $\boldsymbol{z}()$ respect to a component $y_{l}$ of vector $\boldsymbol{y}$, analytical approach requires evaluation of $\operatorname{Jac}(\varphi(c))$ deriving the loading function respect to the components of vector $\mathbf{y}$, and an assignment procedure is needed. Numerical approach requires two values of the function $z()\left(z\left(y_{l}\right)\right.$ and $z\left(y_{l}+h\right)$, being $h$ the increment), needing another assignment procedure. Moreover, if it chooses an approach with central derivatives, the function must be evaluated in the points $\left(y_{l}-h / 2\right)$ and $(y+h / 2)$ and it needs to do two assignment procedures.

In conclusion, the numerical approach requires at least twice the assignment procedures. 
Considering the computation time, the analytical could be about twice as fast as numerical.

Table 1: Designed values at junctions.

\begin{tabular}{ccc}
\hline Node & Link & $y^{*}$ \\
\hline 1090 & $1004-1090$ & 0.41 \\
& $1092-1090$ & 0.59 \\
1044 & $1043-1044$ & 0.40 \\
& $1040-1044$ & 0.60 \\
1047 & $1046-1047$ & 0.39 \\
& $1044-1047$ & 0.61 \\
1048 & $1047-1048$ & 0.40 \\
& $1051-1048$ & 0.60 \\
1053 & $1045-1048$ & 0.60 \\
& $1048-1053$ & 0.39 \\
1026 & $1052-1053$ & 0.61 \\
& $1062-1026$ & 0.26 \\
1062 & $1079-1026$ & 0.74 \\
& $1065-1062$ & 0.25 \\
1046 & $1112-1062$ & 0.75 \\
& $1090-1046$ & 0.40 \\
& $1043-1046$ & 0.60 \\
1065 & $1049-1046$ & 0.60 \\
\hline
\end{tabular}

\section{Conclusions}

In this paper, an analytical method to design the signal at junctions whit the gradient projection algorithm is applied in a real network. The method evaluates the gradient in a closed form; the constraint is to evaluate the path choice probability with a logit model. Particularly, an approach by means of C-Logit model is applied, considering the overlap among different paths.

The proposed method is applied in the network of the city of Reggio Calabria to design some junctions. The obtained results are discussed, observing the improvement in the time spent in the network by the users and in energy consumption.

One of the advantages of the proposed method consists on the computing speed, which makes the procedure usable for the real-time optimization also with real dimension network for the reduction of the energy consumption in urban area.

Improvements at this work are related to: a more robust definition of the connection between the analytically evaluation of the derivatives in the gradient method and the adopted path choice model; a new algorithm to find the descent direction in gradient algorithm. 


\section{References}

[1] Gallo, M. \& D'Acierno, L., Comparing Algorithms for Solving the Local Optimisation of the Signal Settings (LOSS) Problem under Different Supply and Demand Configurations, Procedia - Social and Behavioral Sciences, 87, pp. 147-162, 2013.

[2] Improta, G. \& Cantarella, G.E., Control systems design for an individual signalised junction. Transportation Research 18B, 147-167, 1984.

[3] Webster, F.V. \& Cobbe, B.M., Traffic Signals. Road Research Technical Paper No. 56, London UK, 1966.

[4] Cascetta, E., Gallo, M. \& Montella, B., Optimal signal setting on traffic networks with stochastic equilibrium assignment. Proceedings of TRISTAN III, Puerto Rico, pp. 17-23, 1998.

[5] Gartner, N.H., Area traffic control and network equilibrium, Traffic Equilibrium Methods, Lecture Notes in Economics and Mathematical Systems, 118, M. Florian, ed., Springer-Verlag, Berlin, 274-297, 1976.

[6] Little, J.D.C., Kelson, M.D. \& Gartner, N.H., MAXBAND: A program for setting signals on arteries and triangular networks. Transportation Research Record 795, 40-46, 1981.

[7] Robertson, D.I., TRANSYT method for area traffic control. Traffic Engineering \& Control, 10, 276-281, 1969.

[8] Cantarella, G.E., Improta, A. \& Sforza, A., A procedure for equilibrium network traffic signal setting. Transportation Research, 25 A, pp. 241-249, 1991.

[9] Cantarella, G.E., Velonà, P. \& Vitetta, A., Signal setting with demand assignment: global optimization with day-to-day dynamic stability constraints. Journal of advanced transportation 46, pp. 254-268, 2012.

[10] Marcianò, F. A., Musolino, G. \& Vitetta, A., Signal setting optimisation with route choice behaviour and acyclic profiles of vehicular flows in road networks. Procedia - Social and Behavioral Sciences, 54(4), pp. 1330-1338, 2012.

[11] Varia, H.R., Gundaliya, P.J. \& Dhingra, S.L., Application of genetic algorithms for joint optimization of signal setting parameters and dynamic traffic assignment for the real network data. Research in Transportation Economics, 38, pp. 35-44, 2013.

[12] Ghatee, M. \& Hashemi, S. M., Descent direction algorithm with multicommodity flow problem for signal optimization and traffic assignment jointly, Applied Mathematics and Computation, 188, pp. 555-566, 2007.

[13] Marcianò, F. A., Musolino, G. \& Vitetta A., Signal setting design on a road network: Application of a system of models in evacuation conditions, WIT Transactions on Information and Communication Technologies, 43, pp. PI443-PI454, 2010.

[14] Marcianò, F. A., Musolino, G. \& Vitetta, A., A system of models for signal setting design of a signalized road network in evacuation conditions, WIT Transactions on the Built Environment, 111, pp. 313-323, 2010. 
[15] Polimeni, A. \& Vitetta, A., Joint network and route optimization in road evacuation, WIT Transactions on Ecology and the Environment, 155, pp. 1053-1065, 2011.

[16] EMEP-EEA air pollutant emission inventory guidebook 2013, http://www.eea.europa.eu//publications/emep-eea-guidebook-2013 Last access: 13/06/2014

[17] Cascetta, E., Nuzzolo, A., Russo, F. \& Vitetta, A. (1996), A modified logit route choice model overcoming path overlapping problems, specification and some calibration results for interurban networks. Lesort J.B. (ed.) Transportation and Traffic Theory, pp. 697-711, Pergamon, 1996.

[18] Cirianni, F., Panuccio, P. \& Rindone, C., A comparison of urban planning systems between the UK and Italy: commercial development and city logistic plan, WIT Transactions on the Built Environment, 130, pp. 785-797, 2013.

[19] Cascetta, E., Transportation systems engineering: theory and methods. Kluwer Academic Publisher, 2001.

[20] Pavone, G., Progetto dell'offerta dei sistemi di trasporto stradale urbano, $\mathrm{PhD}$ thesis, 2005. 